1. Roger P, et al. Dissociated overexpression of cathep$\sin \mathrm{D}$ and estrogen receptor alpha in preinvasive mammary tumors. Hum Pathol. 2000;31(5):593-600.

2. Shaaban AM, et al. Declining estrogen receptorbeta expression defines malignant progression of human breast neoplasia. Am J Surg Pathol. 2003;27(12):1502-1512.

3. Thomas C, Gustafsson JA. The different roles of ER subtypes in cancer biology and therapy. Nat Rev Cancer. 2011;11(8):597-608.

4. Kuiper GG, Enmark E, Pelto-Huikko M, Nilsson S, Gustafsson JA. Cloning of a novel receptor expressed in rat prostate and ovary. Proc Natl Acad SciU S A. 1996;93(12):5925-5930.

5. Walter P, et al. Cloning of the human estrogen receptor cDNA. Proc Natl Acad Sci U S A. 1985; 82(23):7889-7893.

6. Heldring $\mathrm{N}$, et al. Estrogen receptors: how do they signal and what are their targets. Physiol Rev. 2007;87(3):905-931.

7. Musgrove EA, Sutherland RL. Biological determi- nants of endocrine resistance in breast cancer. Nat Rev Cancer. 2009;9(9):631-643.

8. Honma N, et al. Clinical importance of estrogen receptor-beta evaluation in breast cancer patients treated with adjuvant tamoxifen therapy. $J$ Clin Oncol. 2008;26(22):3727-3734.

9. Marotti JD, Collins LC, Hu R, Tamimi RM. Estrogen receptor-beta expression in invasive breast cancer in relation to molecular phenotype: results from the Nurses' Health Study. Mod Pathol. 2010;23(2):197-204.

10. Cheng $\mathrm{L}$, et al. PES1 promotes breast cancer by differentially regulating ER $\alpha$ and ER $\beta$. J Clin Invest. 2012;122(8):2857-2870.

11. Li J, et al. Down-regulation of pescadillo inhibits proliferation and tumorigenicity of breast cancer cells. Cancer Sci. 2009;100(12):2255-2260.

12. Maiorana A, Tu X, Cheng G, Baserga R. Role of pescadillo in the transformation and immortalization of mammalian cells. Oncogene. 2004;23(42):7116-7124.

13. Prisco M, et al. Role of pescadillo and upstream binding factor in the proliferation and differentiation of murine myeloid cells. Mol Cell Biol. 2004;24(12):5421-5433.

14. Mak P, et al. ERbeta impedes prostate cancer EMT by destabilizing HIF-1alpha and inhibiting VEGFmediated snail nuclear localization: implications for Gleason grading. Cancer Cell. 2010;17(4):319-332.

15. Hurtado A, et al. Regulation of ERBB2 by oestrogen receptor-PAX2 determines response to tamoxifen. Nature. 2008;456(7222):663-693.

16. Lindberg K, Helguero LA, Omoto Y, Gustafsson JA, Haldosen LA. Estrogen receptor beta represses Akt signaling in breast cancer cells via downregulation of HER2/HER3 and upregulation of PTEN: implications for tamoxifen sensitivity. Breast Cancer Res. 2011;13(2):R43

17. Wong NA, Malcomson RD, Jodrell DI, Groome NP, Harrison DJ, Saunders PT. ERbeta isoform expression in colorectal carcinoma: an in vivo and in vitro study of clinicopathological and molecular correlates. J Pathol. 2005;207(1):53-60.

\title{
miR-122 regulates hepatic lipid metabolism and tumor suppression
}

\author{
Jessica Wen and Joshua R. Friedman
}

Division of Gastroenterology, Hepatology, and Nutrition, Department of Pediatrics, Perelman School of Medicine at the University of Pennsylvania, The Children's Hospital of Philadelphia, Philadelphia, Pennsylvania, USA.

\begin{abstract}
In this issue of JCI, two independent groups describe the effects of germline and liver-specific deletion of Mir122a, the predominant liver miRNA. Their findings reveal a critical role for miR-122 in fat and cholesterol metabolism but suggest that other metabolic actions of the liver are independent of miR122. Knockout mice also displayed hepatic inflammation, fibrosis, and a high incidence of hepatocellular carcinoma, suggesting that miR-122 has a tumor suppressor role in hepatocytes.
\end{abstract}

MicroRNAs (miRNAs) are small, noncoding RNA molecules that regulate the expression of complementary messenger RNAs. Since their initial discovery in 1993 in Caenorhabditis elegans, more than 1,400 miRNAs have been detected in the human transcriptome. In addition to regulating physiologic processes, miRNAs have also been implicated in numerous disease states. The broad function of miRNA in the liver was investigated by studying mice with conditional deletion of Dicer1 in hepatocytes $(1,2)$. Despite the lack of mature miRNA in this model, the liver was able to perform the essential functions of blood glucose regulation, albumin production, and bilirubin metabolism. However, over time, it became clear that miRNA plays an important role

Conflict of interest: The authors have declared that no conflict of interest exists.

Citation for this article: J Clin Invest. 2012; 122(8):2773-2776. doi:10.1172/JCI63966. in fat metabolism, inflammation, and cell cycle regulation in the liver, as these animals developed progressive hepatic steatosis, hepatitis, apoptosis, and hepatocellular carcinoma (HCC) $(1,2)$.

miR-122 is the predominant liver miRNA, making up $70 \%$ of the total miRNA population (3). The activity of miR-122 has previously been assessed through antisense oligonucleotide-mediated knockdown, implicating miR-122 in cholesterol and fat metabolism $(4,5)$. Although HCC was not observed in the time frame of these studies, several groups have reported tumor suppressor activity for miR-122, based on decreased miR122 levels in tumor tissue and inhibitory effects of miR-122 in tumorigenicity assays (6). However, knockdown experiments are limited by their transient nature and the potential for off-target effects.

In this issue of JCI, Hsu et al. and Tsai et al. present definitive evidence of miR-122 function using genetic deletion in mice $(7,8)$. Mice with germline or conditional deletion of Mir122a in the liver were viable and fertile. However, as the animals aged, they developed steatohepatitis, liver fibrosis, and HCC. These groups define direct roles for miR-122 in both fat metabolism and tumor suppression, although it is less clear whether the link to fibrosis is directly or indirectly related to miR-122 loss. Thus, although miR-122 cannot be construed as a "master regulator" of liver function - as the mutant mice have generally normal liver function - it is a critical checkpoint both in hepatic fat production and hepatocellular proliferation (Figure 1).

\section{miR-122 regulates fat and cholesterol metabolism}

Temporary miR-122 inhibition has been shown to reduce serum cholesterol via downregulation of genes involved in cholesterol biosynthesis such as HMG-CoA reductase (4). This is recapitulated in the genetic models: the serum lipid profiles of both liver-specific knockouts (LKO) and germline knockouts (KO) show a 30\% reduction in total cholesterol, LDL, HDL, and serum triglyceride (TG). However, the livers of both $\mathrm{KO}$ and LKO mice also have progressive steatohepatitis $(7,8)$, a feature 


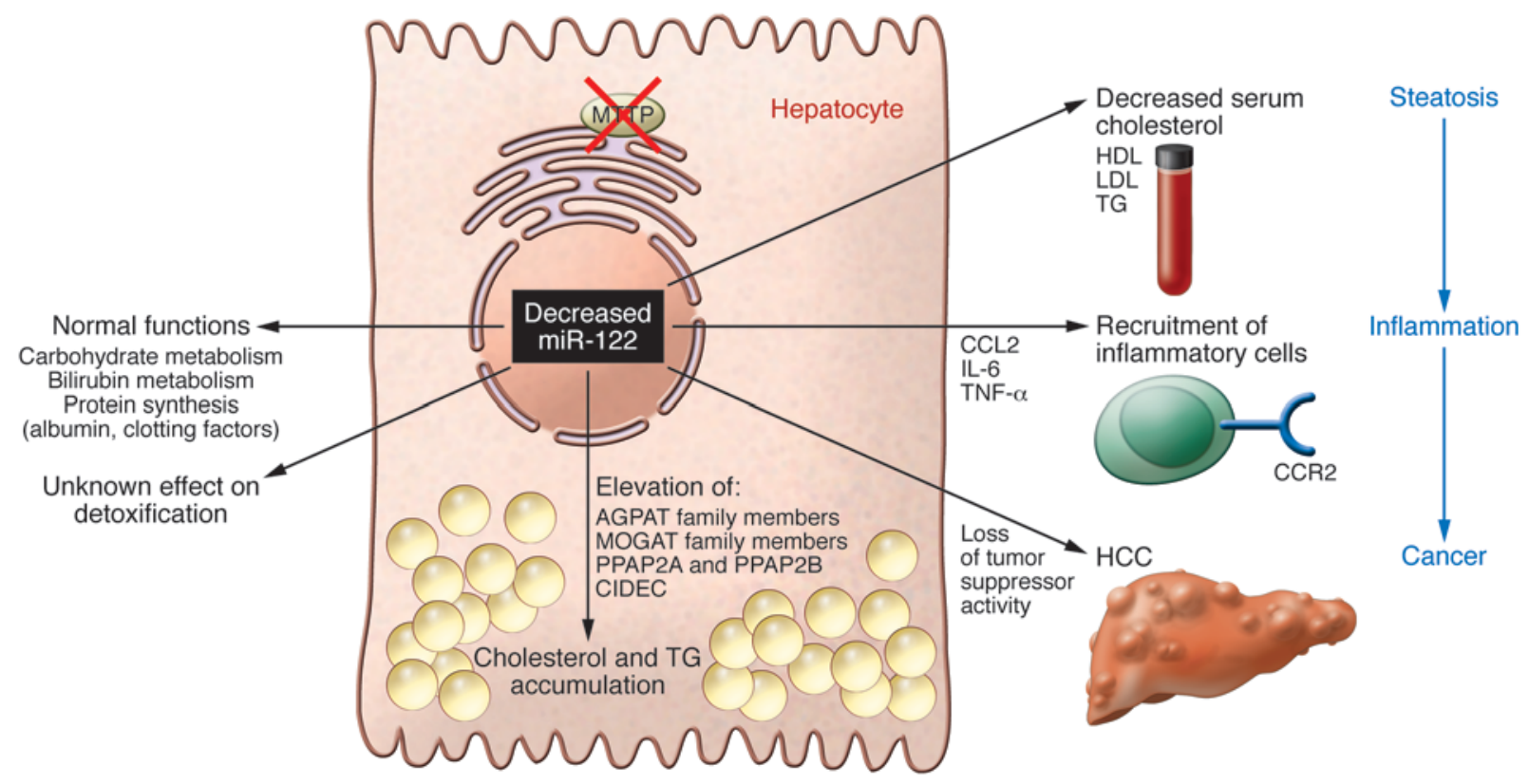

Figure 1

Overview of the consequences of miR-122 loss on hepatocyte function. The normal functions of the hepatocyte include carbohydrate and lipid metabolism, bilirubin excretion, and detoxification of endogenous compounds and xenobiotics. In this issue, Tsai et al. and Hsu et al. demonstrate that loss of miR-122 results in increased lipid synthesis and decreased lipid export, but other hepatocyte functions are unaltered. Loss of miR-122 also led to increased inflammation and fibrosis, and eventually the development of HCC, suggesting the miR-122 plays a tumor-suppressive role.

not seen in the previous knockdown studies $(4,5)$. The concordance of phenotypes in the KO and LKO mice is as expected, given the lack of miR-122 expression in non-hepatic tissues.

Hsu and colleagues identified the mechanism of steatosis in the mutants by performing gene expression profiling of Mir122a-LKO hepatocytes (7). They noted upregulation of genes known to be involved in lipid synthesis in the liver, including Agpat1, Mogat1, Agpat3, Agpat9, Ppap2a, Ppap2c, which are part of the TG biosynthesis pathway (9). Additionally, there was upregulation of Cidec, also known as FSP27, which is a protein that localizes to lipid droplets, negatively regulates lipolysis, and enhances TG accumulation. Consistent with the gene expression changes, the mutant livers synthesize more TG than controls but secrete less, resulting in TG accumulation in hepatocytes. The authors used reporter assays to confirm that Agpat1 and Cidec are direct miR-122 targets.

In their analysis of the fatty liver phenotype, Tsai and colleagues focused on MTTP (microsomal TG transfer protein) and found its expression level to be reduced in the Mir122a-KO mice (8). MTTP normally functions by enhancing the rate of lipid transfer between vesicles and is necessary for the assembly of lipoproteins (10). When Mttp expression was restored, serum cholesterol and fasting TG levels normalized. This surprising result suggests that either the lipid phenotype of the $\mathrm{KO}$ mice is primarily due to effects on Mttp expression or that overexpression of Mttp has a dominant effect on hepatic steatosis. The authors note that Mttp is not predicted to be a direct target of miR-122; this makes sense in light of the parallel loss of $M t t p$ in mice lacking the repressive activity of miR-122. While Hsu and colleagues do not present any data regarding Mttp, it is noteworthy that they did detect a decrease in the expression of another microsomal lipid transporter, Stard4 (7).

Based on the these reports, miR-122 appears to play an important role in cholesterol and TG metabolism within the liver by directly or indirectly regulating cholesterol and TG biosynthesis and excretion. They make it clear that a decrease in circulating TG is not beneficial if the underlying reason is excessive retention of TG within hepatocytes. Likewise, the decrease in serum lipoproteins is of interest from the cardiovascular standpoint, although both the LDL and the HDL fractions were depressed; it will be important for future studies to determine the effect miR-122 loss in the context of atherosclerosis.

\section{miR-122 loss results in hepatic inflammation and fibrosis}

Mir122a-KO animals develop steatohepatitis and liver fibrosis $(7,8)$, phenotypes that were not observed in prior studies using antisense oligonucleotide-mediated Mir122a knockdown. Both groups also demonstrated an increase in infiltrating inflammatory cells in miR-122-deficient liver $(7,8)$. The infiltrating cells were CD $11 b^{\text {hi }} \mathrm{Gr}^{+}$, a population of immature myeloid cells with monocyte and granulocyte morphology. They produce high levels of IL- 6 and TNF- $\alpha$, and these cells are known to promote fibrosis in the injured liver (11).

Is inflammation directly regulated by miR-122, or is the phenotype an indirect result of chronic steatosis? The experiment employing exogenous restoration of Mttp expression implies the latter, because resolution of steatosis by $M t t p$ also led to reduced inflammation and fibrosis. On the other hand, the authors used reporter assays to show that the chemokine gene $\mathrm{Ccl} 2$ is a direct miR-122 target gene in Hepa cells, albeit only to a mild degree (8).

The Mttp rescue experiment also implies that miR-122 regulates hepatic fibrosis indirectly. However, Klf6 was identified as a miR-122 target gene in reporter assays, 
and the suppression of Klf6 by shRNA led to a reduction in TGF- $\beta 1$ expression and collagen deposition in the $\mathrm{KO}$ mice (8). Nevertheless, the reporter assays revealed a less than 2-fold effect of miR-122 on Ccl2 and Klf6 expression. Finally, the reduction in fibrosis observed following treatment with Klf6 shRNA may be completely independent of miR-122 function, because KLF6 is also expressed in activated hepatic stellate cells (which lack miR-122), where it regulates many fibrosis genes (12). Thus, the most likely sequence of events in these models is that steatosis is caused by direct effects of miR-122 deficiency, and that inflammation and fibrosis follow, with minor effects due to de-repression of $\mathrm{Ccl} 2$ and Klf6 in hepatocytes.

\section{miR-122 is a hepatic tumor suppressor}

miR-122 levels are reduced in experimental models and human samples of HCC, and loss of miR-122 is associated with tumor invasiveness and cancer progression (13-15). Therefore, it has been speculated that this miR acts as a tumor suppressor. In the models described by Tsai et al. and Hsu et al., HCC develops in both the KO and LKO mice. In the germline knockout, the incidence is $50 \%$ at 1 year of age. Both groups noted a striking sex disparity in the occurrence of HCC in miR-122-deficient mice, with a male to female ratio of approximately $4: 1$ in the LKO mice (7) and the KO mice (8). Hsu and colleagues did not observe a sex bias in the KO mice they analyzed, but in light of both studies, this may reflect random variation from male predominance. The males also have greater tumor burden and more advanced tumor grade than the females. Both of these features are similar to those observed in human HCC (16). In the mouse model, the sex disparity may be due in part to increased IL- 6 levels in the males, illustrating the complex relationship among sex hormones, inflammation, and tumor suppressors/oncogenes in HCC.

To further understand the involvement of miR-122 in carcinogenesis, Hsu et al. made use of an HCC experimental model wherein transgenic mice harbor both a tetracycline-repressible $M Y C$ gene and a liver activator promoter-driven tet-transactivator protein, resulting in hepatic tumors in the absence of liver damage or inflammation. Interestingly, miR-122 levels are strongly reduced in this model. Remark- ably, administration of a recombinant adeno-associated viral vector expressing MIR122 (after the establishment of small tumors) resulted in a reduction in tumor burden from $40 \%$ to $7.7 \%$ of liver mass. Thus, miR-122 appears to act as a tumor suppressor in a manner that is independent of its roles in fat metabolism or inflammation. It likely mediates this effect via a variety of target genes that are important in cell cycle regulation and hepatocyte differentiation, such as the stem cell genes Prom1, Thy1, and Epcam. To further support a direct tumor suppressor function for miR-122, it would be interesting to determine whether the restoration of Mttp also prevents HCC in the KO mice, a question not addressed by Tsai and colleagues (8). Finally, miR-122 loss may also promote epithelial-mesenchymal transition, as E-cadherin mRNA levels were reduced in the mutant livers even before the appearance of HCC.

\section{Other functions of miR-122}

A recent study has linked miR-122 to liver development through a positive feedback loop with the Onecut hepatocyte transcription factor gene family (17). Mouse embryos lacking both Onecut1 (Hnf6) and Onecut 2 have reduced hepatic miR-122 levels, and Onecut 1 can bind and activate the Mir122a locus. Transgenic mice overexpressing miR-122 in the developing liver have elevated Onecut1 levels and subtle defects in hepatocyte and cholangiocyte differentiation. However, lossof-function analysis in this study was restricted to morpholino-based knockdown in zebrafish, with the analysis limited to early time points. In this respect, the Mir122a-KO mouse has the potential to confirm a developmental function for miR-122. Hsu et al. noted that liver histology was normal at birth and that Onecut1 expression was not altered in adult LKO or KO mice. Although not described in either of the articles, it will be revealing to analyze Onecut1 levels and liver cell differentiation at embryonic time points in the mutant mice; based on the Mir122a-transgenic mouse (17), there may be defects in liver cell differentiation or developmental timing.

miR-122 also has a compelling function in HCV replication. It is required for viral replication and interacts with HCV RNA, although it is not required for HCV RNA synthesis (18-21). Although miR-122 levels have not been directly correlated with viral load in liver samples from patients infected with HCV (22), in non-human primates, treatment with a miR-122 inhibitor results in a significant decrease in HCV viral load, and it is being investigated as a potential therapeutic for $\mathrm{HCV}(23)$. The two miR-122 studies in this issue of the JCI suggest that the use of this miR as anti-HCV therapy will need to be balanced with the potential for developing HCC, especially since patients with chronic HCV already have an increased risk for HCC. Furthermore, patients with hepatitis $\mathrm{C}$ have increased risk for cardiovascular disease and insulin resistance, so the effects of miR-122 inhibition on cardiovascular health will also need to be considered.

As a whole, the findings reported by Hsu et al. and Tsai et al. definitively describe the phenotype of miR-122 loss in the quiescent liver. miR-122 is a key regulator of multiple hepatic pathways, as highlighted by its direct roles in fat metabolism, tumor suppression, and perhaps inflammation and fibrosis. Despite this, liver development and hepatocyte differentiation occur normally in mice lacking miR-122. miR-122-deficient livers also continue to perform bilirubin, protein, glucose, and xenobiotic metabolism. In future studies, it will be intriguing to see whether these other functions are dependent on miR-122 in the context of stress, such as a high-fat diet or hepatotoxins. Conversely, while loss of miR-122 causes steatohepatitis, it is unknown whether exogenous miR-122 can ameliorate fatty liver disease causes by diet or toxins. Finally, the tumor-suppressive function of miR-122 illustrated by both groups suggests that the restoration of miR-122 expression may be of benefit in HCC - but as with many other tumor suppressor miRNAs, delivery of the miRNA remains a major challenge.

\section{Acknowledgments}

The authors wish to thank the Fred and Suzanne Biesecker Pediatric Liver Center for support of this work.

Address correspondence to: Joshua R. Friedman, Department of Pediatrics, Division of Gastroenterology, Hepatology, and Nutrition, Perelman School of Medicine at the University of Pennsylvania, The Children's Hospital of Philadelphia, 3615 Civic Center Blvd., ARC 902G, Philadelphia, Pennsylvania 19104, USA. Phone: 267.426.7223; Fax: 206.984.2191; E-mail: friedmaj@mail.med.upenn.edu. 
1. Hand NJ, Master ZR, Le Lay J, Friedman JR. Hepatic function is preserved in the absence of mature microRNAs. Hepatology. 2009;49(2):618-626

2. Sekine S, et al. Disruption of Dicer1 induces dysregulated fetal gene expression and promotes hepatocarcinogenesis. Gastroenterology. 2009;136(7):2304-2315.e4.

3. Lagos-Quintana M, Rauhut R, Yalcin A, Meyer J, Lendeckel W, Tuschl T. Identification of tissue-specific microRNAs from mouse. Curr Biol. 2002;12(9):735-739.

4. Esau C, et al. miR-122 regulation of lipid metabolism revealed by in vivo antisense targeting. Cell Metab. 2006;3(2):87-98.

5. Krutzfeldt J, et al. Silencing of microRNAs in vivo with 'antagomirs'. Nature. 2005;438(7068):685-689.

6. Saito Y, et al. MicroRNAs in hepatobiliary and pancreatic cancers. Front Genet. 2011;2:66

7. Hsu S-H, et al. Essential metabolic, anti-inflammatory, and anti-tumorigenic functions of miR-122 in liver. J Clin Invest. 2012;122(8):2871-2883.

8. Tsai W-C, et al. MicroRNA-122 plays a critical role in liver homeostasis and hepatocarcinogenesis. J Clin Invest. 2012;122(8):2884-2897.

9. Kim YJ, Cho SY, Yun CH, Moon YS, Lee TR, Kim $\mathrm{SH}$. Transcriptional activation of Cidec by PPAR- gamma2 in adipocyte. Biochem Biophys Res Commun. 2008;377(1):297-302.

10. Hussain MM, Shi J, Dreizen P. Microsomal triglyceride transfer protein and its role in apoB-lipoprotein assembly. J Lipid Res. 2003;44(1):22-32.

11. Karlmark KR, et al. Hepatic recruitment of the inflammatory Gr1+ monocyte subset upon liver injury promotes hepatic fibrosis. Hepatology. 2009;50(1):261-274.

12. Miele L, et al. The Kruppel-like factor 6 genotype is associated with fibrosis in nonalcoholic fatty liver disease. Gastroenterology 2008;135(1):282-291.e1.

13. Wu X, et al. miR-122 affects the viability and apoptosis of hepatocellular carcinoma cells. Scand J Gastroenterol. 2009;44(11):1332-1339.

14. Coulouarn C, Factor VM, Andersen JB, Durkin ME, Thorgeirsson SS. Loss of miR-122 expression in liver cancer correlates with suppression of the hepatic phenotype and gain of metastatic properties. Oncogene. 2009;28(40):3526-3536.

15. Bai S, et al. MicroRNA-122 inhibits tumorigenic properties of hepatocellular carcinoma cells and sensitizes these cells to sorafenib. J Biol Chem. 2009;284(46):32015-32027.

16. McGlynn KA, London WT. Epidemiology and natural history of hepatocellular carcinoma. Best Pract
Res Clin Gastroenterol. 2005;19(1):3-23.

17. Laudadio I, et al. A feedback loop between the liverenriched transcription factor network and miR122 controls hepatocyte differentiation. Gastroenterology. 2012;142(1):119-129.

18. Machlin ES, Sarnow P, Sagan SM. Masking the $5^{\prime}$ terminal nucleotides of the hepatitis $\mathrm{C}$ virus genome by an unconventional microRNA-target RNA complex. Proc Natl Acad Sci U S A. 2011;108(8):3193-3198.

19. Nunnari G, Schnell MJ. MicroRNA-122: a therapeutic target for hepatitis $\mathrm{C}$ virus (HCV) infection. Front Biosci (Schol Ed). 2011;3:1032-1037.

20. Jopling CL. Regulation of hepatitis C virus by microRNA-122. Biochem Soc Trans. 2008; 36(Pt 6):1220-1223

21. Shimakami T, et al. Stabilization of hepatitis C virus RNA by an Ago2-miR-122 complex. Proc Natl Acad Sci US A. 2012;109(3):941-946.

22. Sarasin-Filipowicz M, Krol J, Markiewicz I, Heim $\mathrm{MH}$, Filipowicz W. Decreased levels of microRNA miR-122 in individuals with hepatitis C responding poorly to interferon therapy. Nat Med. 2009;15(1):31-33.

23. Lanford RE, et al. Therapeutic silencing of microRNA-122 in primates with chronic hepatitis C virus infection. Science. 2010;327(5962):198-201.

\title{
How many neutrophils are enough (redux, redux)?
}

\author{
Samuel C. Silverstein ${ }^{1,2}$ and Raul Rabadan 3,4
}

1Department of Physiology and Cellular Biophysics, ${ }^{2}$ Department of Medicine, ${ }^{3}$ Department of Biomedical Informatics, and ${ }^{4}$ Center for Computational Biology and Bioinformatics, College of Physicians and Surgeons, Columbia University, New York, New York, USA.

\begin{abstract}
Many chemotherapeutic regimens produce neutropenia, which predisposes to microbial infection. However, not all neutropenic individuals develop infections, so the ability to predict this outcome would be a powerful clinical tool. In this issue of the JCI, Malka et al. describe a dynamic system model of neutrophil bactericidal activity that confirms and extends the concept of critical neutrophil concentration. The authors demonstrate that when the neutrophil concentration approaches the critical concentration, bacterial populations in contact with them exhibit bistability. Their experimental findings raise the intriguing possibility of greater variability in bactericidal activity of neutrophils from healthy adults than heretofore recognized; their model predicts that this could have life-and-death consequences.
\end{abstract}

Although the link between neutropenia and infection risk is clear, the precise number of neutrophils required to maintain health remains a topic of intense research interest. Neutrophils continuously patrol the luminal surfaces of endothelial cells, searching for signs of infection or inflammation. Such signs stimulate them to emigrate from blood into extravascular compartments (1). There, their armamen-

Conflict of interest: Raul Rabadan is a consultant for Novartis and the Geneva Foundation. Samuel C. Silverstein owns stock in Roche Pharmaceuticals and Teva Pharmaceuticals.

Citation for this article: J Clin Invest. 2012; 122(8):2776-2779. doi:10.1172/JCI63939. tarium of chemoattractant and phagocytosis-promoting plasma membrane opsonin and pattern-recognition receptors enable them to phagocytose approximately 40-50 bacteria/neutrophil (2), and their preformed granule proteins and capacity to produce high intravacuolar concentrations of reactive oxygen species (3) enable them to kill their bacterial prey. The extracellular release of DNA-histone antimicrobial protein-containing nets (4) that entrap bacteria and kill yeast/fungi (5) extends their bactericidal and fungicidal activities into their afterlives and further enhances their efficacy as guardians of tissue sterility.
Chemotherapeutic agents that inhibit neutrophil biogenesis (6-8) and/or reduce their bactericidal activity (7) produce neutropenia (i.e., <500,000 neutrophils/ml blood). Although neutropenia predisposes to infection, it has little direct effect on the sterility of blood because under most (9) but not all (10) - conditions, hepatic and splenic macrophages are the cells primarily responsible for clearing bacteria from the circulation. Indeed, as suggested by Crosby (11) and supported by the studies of Wright et al. (12) and Koene et al. (13), it is the tissue neutrophil concentration $(N)$ that determines whether the small number of bacteria that breech mucosal surfaces each day will find fertile soil for growth, or be engulfed and killed. Thus, while a blood $N$ value of $5 \times 10^{5}$ neutrophils $/ \mathrm{ml}$ is a call for vigilance, it is an imprecise measure of the likelihood of infection. This is so because blood is primarily the conduit by which neutrophils travel from bone marrow to tissues, and the blood $N$ reflects the sum of the rates at which neutrophils are produced and released from bone marrow into the circulation, and the rates at which they are consumed in tissues (14) and/or recycled to spleen and bone marrow for destruction (15). 